\title{
The Effect of the Concentration Polarization and the Membrane Layer Mass Transport on the Membrane Separation
}

\author{
Endre Nagy ${ }^{1 *}$ \& Gábor Borbély ${ }^{2}$ \\ ${ }^{182}$ University of Pannonia, Research Institute of Chemical and Process Engineering, P.O. Box 158, 8201 \\ Veszprém, Hungary
}

\begin{abstract}
The negative effect of the concentration polarization layer on the membrane separation is well known. How the mass transport parameters of the membrane matrix, e.g. the solubility coefficient, membrane Peclet number, can affect the concentration profile of the boundary layer, and consequently, the separation efficiency is not investigated in detail yet. This paper gives the suitable mathematical expressions, in order to predict the well known parameters as polarization modulus, enrichment factors, etc., taking into account the transport parameters for both the concentration boundary and the membrane layers, and analyses the concentration distribution and the polarization modulus. It has been shown that the transport properties of the membrane layer have significant effect on the concentration profiles of the boundary layer and thus, on the polarization modulus, enrichment factors, etc., as well. Thus, the well known equations, e.g. the polarization modulus, enrichment factor given in the literature [see e.g. Equations (2) and (3)], could be considered as approaches.
\end{abstract}

Keywords: Concentration, polarization, membrane mass transport, membrane separation, enrichment factor, polarization modulus

\subsection{INTRODUCTION}

The negative effect of the stagnant concentration boundary layer on the mass transport through a membrane has been investigated for a long time. It was proved that this layer can be a major limiting factor in various membrane separation processes [1]. Two main types of the concentration polarization layers can be distinguished, namely the membrane separation can lead to an accumulation of the retained species (Figure $1(\mathrm{a})$ ) or a depletion of the preferentially permeating components in the boundary layer due to its permeation through the membrane, adjacent to it (Figure 1(b)). The polarization can essentially reduce the overall efficiency of separation, it can severely limit flux and selectivity. Number of paper analyzed the effect

* Correspondence to: Endre Nagy \& Gábor Borbély (email: nagye@mik.vein.hu, borbély@mukki.richem.hu) of the concentration polarization and defined the well known equation to its prediction [e.g. 1-8]. Almost all earlier studies [1-2] consider the mass transport equations in the boundary layer, only, defining its Peclet number $\left(P e_{L}=v \delta / D_{L}\right)$, but do not discuss that in the membrane layer and its effect back to the concentration polarization layer and on the efficiency of the membrane separation. This can practically mean that the membrane concentration gradient is regarded to be zero as limiting case of the Peclet number, namely $P e_{m} \rightarrow \infty\left(P e_{m}=v \delta_{m} / D_{m}\right)$ as well as that it does not alter the concentration profile in the boundary layer. In the most cases, the diffusion coefficient of transporting species can be 1-2 order of magnitudes lower than that in the continuous liquid phase and consequently, the $P e_{m}>>P e_{L}$ can easily be fulfilled. But, considering the other variables, namely the thickness of the membrane and that of the concentration boundary layer, the value of $\delta_{m}$ can often be much smaller than that 


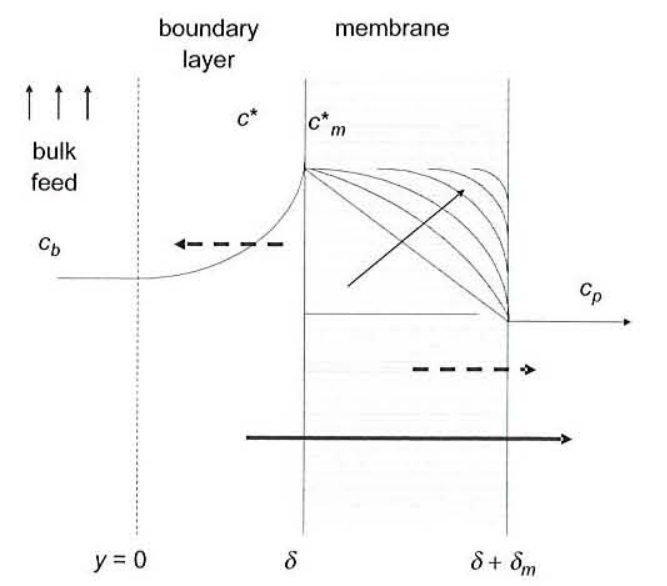

(a)

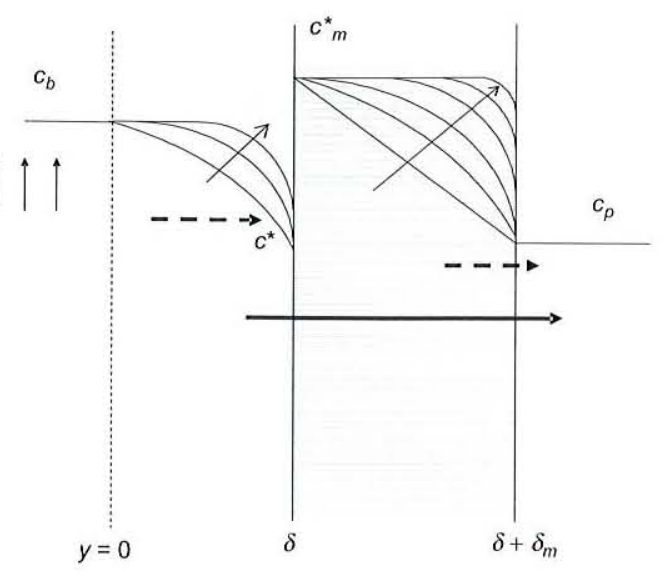

(b)

Figure 1 Concentration profiles in the concentration boundary layer and membrane layer

of the concentration polarization layer, $\delta$. As a consequence the above inequality of Peclet numbers does not fulfill anymore. This fact proves that the effect of the membrane Peclet number on the well known separation parameters (e.g. concentration of the solute at the membrane interface, $C^{*}\left[S_{m} C^{*}=C_{m}^{*}\right]$, concentration polarization modulus, I, enrichment factor, $E$, intrinsic enrichment, $E_{o}$, etc.) should also be discussed and analyzed. This makes possible to get more general equations between the separation parameters which contain the effect of the membrane layer, as well. The method used has also been applied for three-layer mass transport (concentration boundary layer, gel layer and membrane layer) as well (not discussed in this paper).

\subsection{BRIEF REVIEW OF THE PREVIOUS} WORKS

At steady state, the sum of the convectiv (continuous arrows in Figures 1(a) and (b) and diffusive transport (dotted arrows in Figures 1 (a) and (b)) in the boundary layer equals the amount permeated through the membrane:

$$
v C-D \frac{d C}{d y}=v C_{p}
$$

Equation (3) allows the prediction of the of $P e_{L}$ and the function of the intrinsic enrichment

factor, $E_{o}$. The concentration polarization modulus should be larger than unity if the permeating compound is depleted in the membrane (Figure 1(a)) and less than unity if the permeating compound is enriched in the permeate (Figure 1(b)) [4].

The above model does not contain the mass transport properties of the membrane layer. It can be said it is considered as a black box that has no any influence on the concentration of the boundary layer. Baker et al. [4] as well as She and Hwang [7-8] have analyzed the effect of the boundary layer on the separation of dilute flavor organics applying pervaporation process. They combined the concentration polarization equation [Equation (2)] with the solution-diffusion model of the membrane transport, taking into account of the diffusive mass flow $\left(J_{D}\right)$ through the membrane, only. Thus, the membrane Peclet number, $P e_{m}$ is regarded to be infinitely low, i.e. $P e_{m} \rightarrow 0$. If you accept that, according to the $e_{m} \rightarrow 0$. If you accept continuity equation, the convective velocity of the continuous phase in the membrane should be equal to that in the concentration boundary, the assumption of $P e_{m} \rightarrow 0$ is fulfilled only when $\delta / D_{L}>>\delta_{m} / D_{m}$. This inequality is very rarely true to our opinion.

Pressure-driven membrane process as ultrafiltration is widely used for separation of macromolecules or colloidal particles from liquid. In
this case when the permeate flux are larger than this case when the permeate flux are larger than the diffusive flux in reversed direction (dotted line Figure 1(a) in the boundary layer) the macromolecules starts to deposit on the membrane surface building a cake (gel) layer on it $[9,10]$. In this paper the fouling will not be discussed in details. Because of its importance in the separation, it will be discussed in our conference lecture.

\subsection{THEORY}

Applying a composite (or asymmetric) membrane with a very thin active layer, the value of $P e_{m}=v_{L} \delta_{m} / D_{m}$ can be comparable with that in the concentration boundary layer. In this case $d C_{m} / d y \neq 0$, thus, the effect of the $P e$ should also be taken into account. The concentration distributions of the membrane- and concentration polarization layers are illustrated in Figure 1(a) and Figure 1(b). The differential mass balance equation, perpendicular to the membrane interface, for the concentration boundary layer and the membrane layer is, according to Equation (1), is as follows:

$$
v \frac{d C_{j}}{d y}-D_{j} \frac{d^{2} C_{j}}{d y^{2}}=0 \quad \text { with } j=L, m
$$

where indices $L, m$ denote the boundary layer and membrane matrix, respectively. After integration of Equation (4), the concentration distribution of the boundary layer and the membrane layers can be given, respectively, as follows $(Y=y / \delta)$

$C_{L}=T_{L} e^{P e_{L} Y}+Q_{L} \quad 0 \leq Y \leq 1$

$C_{m}=T_{m} e^{\left(P e_{m} \delta / \dot{\delta}_{m}\right) Y}+Q_{m} \quad 1 \leq Y \leq 1+\delta_{m} / \delta \quad$ (6)

The boundary conditions to determine the values of $T_{L}, Q_{L}, T_{m}, Q_{m}$ parameters are as follows:

$T_{L}+Q_{L}=C_{b} \quad Y=0$

$Q_{L}=Q_{m}$

$S_{m}\left(T_{L} e^{P e_{L}}+Q_{L}\right)=T_{m} e^{P e_{m} \delta / \delta_{m}}+Q_{m} Y=1$

$T_{m} e^{P e_{m}\left(1+\delta / \delta_{m}\right)}+Q_{m}=S_{m} C_{p} \quad Y=1+\delta_{m} / \delta \quad(10)$ where $P e_{L}=v \delta / D_{L}$ and $P e_{m}=v \delta_{m} / D_{m}$

The value of $C_{p}$ is here the equilibrium concentration of the component transported, on the downstream side of membrane, thus, $C_{p} \leq C_{b}$ in the case of constant solubility value.

The values of $Q_{L}$ and $Q_{m}$ gives the overall mass transfer rate (the sum of the diffusive and convective flow), as it is given later [Equation 14], while $S_{m}$ denotes the solubility of the transported compound in the membrane layer. If the solubility is negligible then $S_{m}=1$. The values of parameters $T_{L}, Q_{L}, T_{m}, Q_{m}$, in order to predict the concentration distribution in both layers, are listed in the Appendix. Here some important variables, enrichment, $E$ concentration polarization modulus, $I=E / E_{o}$ are given. 
$I \equiv \frac{C^{*}}{C_{b}}=\frac{\left(1-e^{-P e_{m}}\right) e^{P e_{e_{L}}}+S_{m} E e^{-P e_{m}}\left(e^{P e_{L_{L}}}-1\right)}{S_{m} e^{P e_{L_{L}}}-e^{-P e_{m}}+1-S_{m}}$

Equation (14) is valid for both layers. Taking into account the $Q_{L}\left(Q_{L}=Q_{m}\right)$ value in Equation (A2), the mass transfer rate can be expressed as (11a) (important to note that $k_{L} P e_{L}=k_{m} P e_{m}$, where $k_{L}=D / \delta$ or $\left.k_{m}=D_{m} / \delta_{m}\right)$

$I \equiv \frac{C^{*}}{C_{b}}=\frac{\left(1-e^{-P e_{m}}\right) e^{P e_{e_{L}}}}{S_{m} e^{P e_{L}}-e^{-P e_{m}}+1-S_{m}-S_{m} E_{o} e^{-P e_{m}}\left(e^{P e_{L}}-1\right)}$

$$
J=\beta\left(C_{b}-e^{-\left[P e_{L}+P e_{m}\right]} C_{p}\right)
$$

(11b) where

$$
E_{o} \equiv \frac{C_{p}}{C^{*}}=\frac{E\left(S_{m} e^{P e_{\ell_{L}}}-e^{-P e_{m}}+1-S_{m}\right)}{\left(1-e^{-P e_{m}}\right) e^{P e_{e_{L}}}-S_{m} E e^{-P e_{m}}\left(e^{P e_{L_{L}}}-1\right)}
$$

$$
\beta=\frac{k_{L} P e_{L} S_{m}}{S_{m}\left(1-e^{-P e_{L}}\right)+e^{-P e_{L}}\left(1-e^{-P e_{m}}\right)}
$$

Knowing the value of $I$ and $E_{o}$, the enrichment factor $E$ can be calculated from the expression of $E=I E_{o}$ as follows:

$\left.E \equiv \frac{C_{p}}{C_{b}}=\frac{E_{o}\left(1-e^{-P e_{m}}\right) e^{P e_{e_{L}}}}{S_{m} e^{P e_{L}}-e^{-P e_{m}}+1-S_{m}+S_{m} E_{o} e^{-P e_{m}}\left(1-e^{P e_{L}}\right.}\right)$

can be determined for all components transported through a membrane layer.

Applying the value of the mass transfer rate both the concentration polarization modulus $(I)$ and the enrichment factor $(E)$ can be expressed. In order to give the $I$ value, the interface concentration (at $y=\delta$ ) has to be known. Similarly (13) to Equation (15), the mass transfer rate for the where $E=C_{p} / C_{b} ; \quad E_{o}=C_{p} / C^{*} \quad S_{m} C^{*}=C_{m}$

Values of $I$, given by Equations (11a) and (11b), are perfectly different form that given by Equation (3), due to the different boundary conditions. Let us compare Equations (1) and (10). According to Equation (1) $Q_{u}=C_{p}$ while Equation (10) can essentially differ from this equality. Values of the concentration polarization modulus or enrichment factor can easily be obtained by means of Equation (A1) to (A4) and Equation (5) or Equation (6).

\subsection{Mass Transfer Rate}

Knowing the concentration distribution in both the boundary layer and the membrane layer, the overall mass transfer rate, namely the sum of the convective and diffusive flows, can easily be given. Recently Nagy [12] has analyzed the mass transfer rate through catalytic membrane layer He obtained that the overall mass transfer rate can be given as follows:

$$
J=v C-D \frac{d C}{d y}=v Q_{i} \quad \text { with } i=L, m(14)
$$
[12]:

$$
\begin{gathered}
J=\beta_{L}\left(C_{b}-e^{-P e_{L_{L}}} C^{*}\right) \\
\beta_{L}=\frac{k_{L} P e_{L} e^{P e_{e_{L}}}}{e^{P e_{L_{L}}}-1}
\end{gathered}
$$

Mass transfer rates expressed by Equations (15) and (16) are, obviously, equal to each other: Thus, for the values of $E$ and $I$ can be obtained

$$
\begin{gathered}
E=e^{\left(P e_{L}+P e_{m}\right)}\left(1-\frac{J}{\beta C_{b}}\right) \equiv e^{\left(P e_{L}+P e_{m}\right)}\left(1-\frac{J}{J_{\max }}\right) \\
I=e^{P e_{L_{L}}}\left(1-\frac{J}{\beta_{L} C_{b}}\right) \equiv e^{P e_{L_{L}}}\left(1-\frac{J}{J_{\max }}\right)
\end{gathered}
$$

The values of $\beta_{L} C_{b}$ and $\beta_{L} C_{b}$ are the mass ransfer rate in the case when $C_{p}=0$ and $C^{*}=0$, respectively. Thus, their value can be defined as their maximum value, i.e. $J_{\max }=\beta_{L} C_{b}$ and $J_{L \max }=\beta_{L} C_{b}$. The mass transfer rate can be given membrane layer, applying Equation (14)

\subsection{RESULTS AND DISCUSSION}

A few figures will illustrate the effect of the membrane mass transport on the concentration profile in both the boundary layer and membrane layer: It will be shown how strongly can alter the $P e_{\text {. }}$ number the concentration distribution of the number the concentration distribution of the
concentration boundary layer as well, and consequently, the value of the polarization modulus, $I$ [Equations (11a) and (11b)] and the enrichment factors, $E, E_{o}$ [Equations (12) and (13)]. The effect of the membrane Peclet number, $P e_{\text {in }}$ is lllustred in Figure 2. The vertical doted line the figure gives the inner edge of the boundary layer, at $y=\delta$ (here $\delta=\delta_{m}$ ). It is clearly shown that e.g. the value of the concentration on the membrane interface (at $y=\delta$ ), and consequently the value of $I$, strongly depends on the $P e_{m}$ value. Equation (3) does not contain this effect. With he increase of the $P e$ n value, the average the incease of the $P_{e}$ value, the average the overall mass transfer rate, $J$ [Equation (14)] also increases. During the calculation, the relative value of the outlet concentration, $\left(C_{p} / C_{b}\right)$, was chosen to be 0.1 .

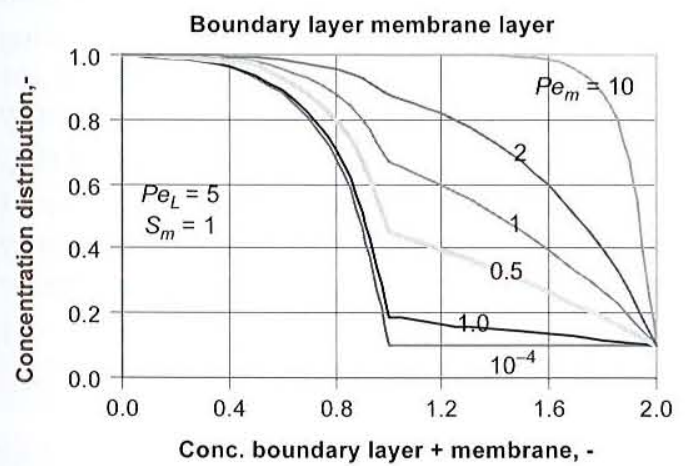

Figure 2 Concentration distribution in the boundary layer and membrane layer at different $P e_{m}$ value (dotted line: $\left.y=\delta, C_{p} / C_{b}=0.1, \delta_{L}=8_{m}\right)$

The effect of the solubility, $S_{m},\left(S_{m} C_{L}=C_{m}\right)$ is also an important parameter (Figure 3). In the case of filtration processes, as ultrafiltration, etc. the solubility could be very often neglected $\left(\mathrm{S}_{m}=1\right)$ or due to the spherical effect $S_{m}<1$

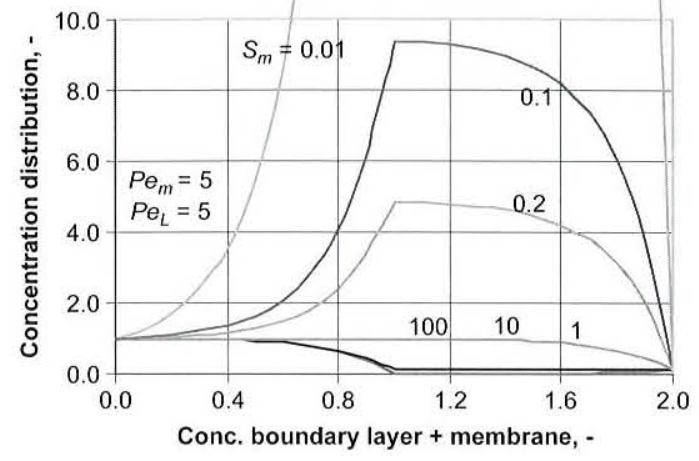

Figure 3 The effect of the solubility, $S_{m}$, on the concentration profiles in the concentration boundary and membrane layer, $C_{p} / C_{b}=0.1, \delta_{L}=\delta_{m}$

(when the particle size and the pore size are close to each other, then the particles, macromolecules, can be hindered in their convective velocity and their solubility, see e.g. [11]). In this case, the well known, convex concentration profile can be formed in the concentration boundary layer as can be seen in Figure 3. When the transported component dissolves in the membrane matrix, hat is $S_{m}>1$, the boundary layer (see lines for $\left.S_{m}=1,10,100\right)$. This is the case for pervaporation where $S_{m}>100$ very often $[4,8,9]$. As can be seen in Figure 3, the concentration on the membrane interface can be very high at low values of $S_{m}$.

Baker et al. [4] plotted $I$ vs. $P e_{L}$ function at different $E_{o}$ values according to Equation (3). In our case, the $E_{o}$ value can't be varied optionally. It is determined by the parameters $\left(P e_{L}, P e_{m}, S_{m}\right)$ and by the inlet and outlet concentrations. These letters determine the value of $E$, namely $E=C_{p} / C_{b}$ proving that this value can be changed freely. This follows alo fiom Equations (A1) to (A4), as well As it cun be sech ing (A4), as well. As it can be seen in Figure 4, the concentration polarization modulus strongly decreases with increasing value of the solubility coefficient. The $I$ value decrease with increasing with values obtained by Equation (3) [4]. When $S_{m}<1$, then the polarization modulus increases with increasing $P e_{L}$. 


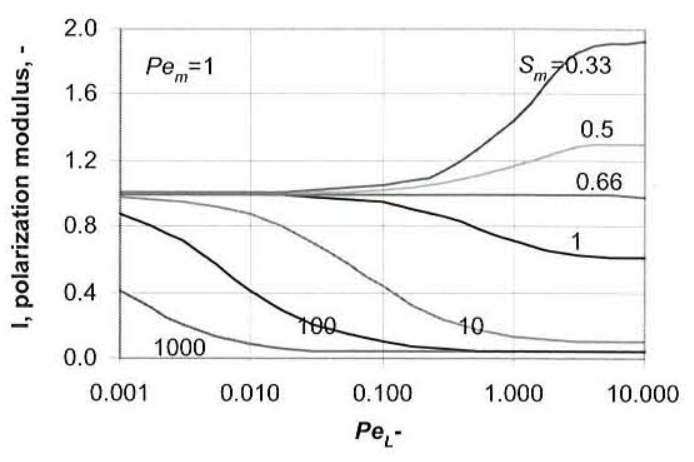

Figure 4 Concentration polarization modulus vs. liquid phase Peclet number, $P e_{L}$, at different values of solubility coefficien $\left(I=C^{*} / C_{b}, C_{p} / C_{b}=0.1, \delta_{L}=\delta_{m}\right)$

The value $P e_{m}$ is an important parameter since it strongly alters the value of the polarization modulus (Figure 5). With increasing $P e_{m}$ value, the polarization modulus also increases. At large values of $P e_{m}$ and $P \mathrm{P}_{\mathrm{L}}$ the value of $I$ tends to unit. The limiting value of $I$, with increasing value of $P e_{L}$ and decreasing value of $P e_{m}$, is $C_{p} / C_{b}=E$.

The above results can easily be applied, as it has been made, for ultrafiltration or for pervaporation processes. The overall mass transfer rate is, probably, the most important quantity of the mass transport. Its value depends on both the $P e_{L}$ and $P e_{m}$ as well as even on the solubility parameter. The $k_{L}$ value $\left(k_{L}=D_{L} / \delta\right)$ alters also separately the value of $J$. How the value of $\beta / k_{L}$

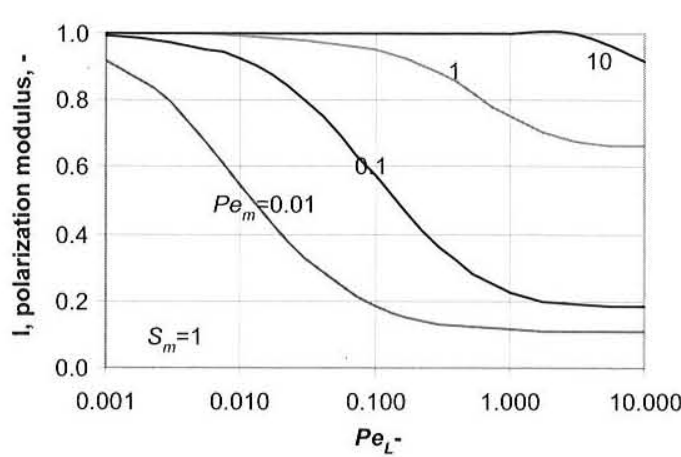

Figure 5 Concentration polarization modulus vs liquid phase Peclet number, $P e_{L}$, at different values of membrane Peclet number $P e_{m}\left(I=C^{*} / C_{b}, C_{p} / C_{b}=0.1, \delta_{L}=\delta_{m}\right)$

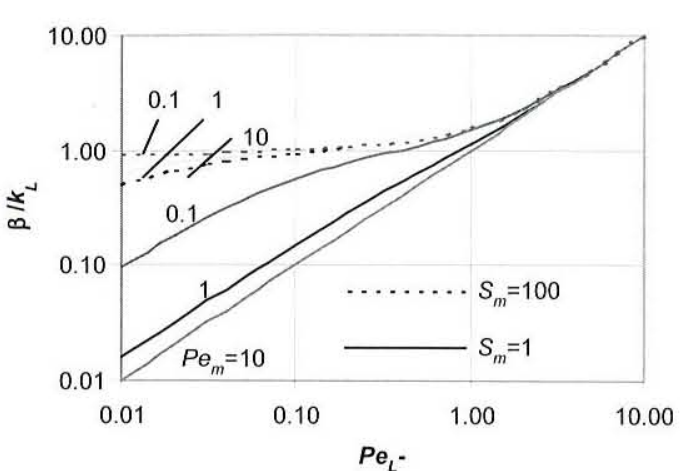

Figure 6 Mass transter enhancement $\left(\beta / k_{L}\right)$ as a function of Peclet-number $\left(P e_{L}\right)$ of the boundary layer at different values of $P e$ and $S_{m}$

changes as a function of $P e_{L}$, is illustrated in Figure 6, at different values of $P e_{m}$ and $S_{m}$. The value of the solubility coefficient can often be larger than 100 , especially during pervaporation process $(4,8,9,13)$. With the increase of the value of $P e_{L}$, the value of $\beta / k_{L}$ tends to a limiting value, namely to the value of $P e_{L}$. It is easy to see from Equation (16) that $\lim \beta / k_{L}=P e_{L}$ if $P e_{L} \rightarrow \infty$. At low values of $P e_{L}, \beta / k_{L}$ depends strongly on both the values of $P e_{2}$ and $S$. If $P e_{L}=P e_{2} \rightarrow 0$ (and $\left.S_{m}=1, D_{L}=D_{m}, \delta=\delta_{m}\right)$ then $\beta / k_{L}=0.5$ proving that only diffusive flow exists and $k_{L}=k_{m}\left(k_{m}=\right.$ $\left.D_{m} / \delta_{m}\right)$. With the decrease of $P e_{L}$ the value of $\beta /$ $k_{L}$ also strongly decreases at a given value of $P e_{m}$ Generally, it can be stated that mass transport through membrane layer, its parameters, namely $P e_{m}$ and $S_{m}$, can strongly alter the mass transfer rate, as wel.

\subsection{CONCLUSION}

The concentration polarization is affected not only by the external hydrodynamic conditions but on the mass transport process inside of the membrane layer. The membrane Peclet number; the solubility coefficient can strongly affect the concentration profile in the concentration boundary layer as well and consequently, the overall mass transfer rate. The traditional models of the concentration polarization does not contain this effect, consequently they can inaccurately describe the mass transport in the concentration polarization layer and, consequently, the separation efficiency. The well known parameters, as polarization modulus, enrichment factor can also be defined by means of the overall mas transfer rate.

\section{ACKNOWLEDGEMEN}

This work was supported by the Hungarian Research Foundation under Grants OTKA $63615 / 2006$

\section{REFERENCES}

[1] Bhattacharya, S., and S.-T. Hwang. 1997 Concentration Polarization, Separation Factor; and Pecl Processes. J. Membrane Sci. 132: 73-90.

[2] Brian, P.L.T. 1966. Mass Transport in Reverse Osmosis, In Desalination by Reverse Osmosis, Edited by U. Merten. Cambridge, USA: M.I.T. 181.

[3] Mulder, M.H.V. 1991. Basic Principles of Membrane Technology. Dordrecht, Kluwe Academic. 416-464.

[4] Baker, R.W., J.G. Wijmans, A.L. Athayde R. Daniels, J.H. Ly, and M. Le. 1997. The Effect of Concentration Polarization on the Separation of Volatile Organic Compounds from Water by Pervaporation. J. Membrane Sci. 137: 159-172.

[5] De, S., and P.K. Bhattacharya. 1997 Modeling of Ultrafiltration Process for Two-component Aqueous Solution of Low and High (gez-forming) Molecular Weight Solutes. J. Membrane Sci. 136: 57-69.

[6] Sablani, S.S., M.F.A. Goosen, R. AlBelushi, and M. Wilf. 2001. Concentration Polarization in Ultrafiltration and Revers Osmosis: A Critical Review. Desalination. 141: $269-289$

[7] She, M., and S.-T. Hwang. 2004. Concentration of Dilute Flavor Compounds by Pervaporation: Permeate Pressure Effect
8] She, M., and S.-T. Hwang. 2006. Effects of Concentration, Temperature, and Coupling on Pervaporation of Dilute Flavor Organics. J. Membrane Sci. 271: 16-28.

[9] Song, L. 1998. A New Model for the Calculation of the Limiting Flux in Ultrafiltration. J. Membrane Sci. 144: 173-185.

[10] Song, L. 1999. Permeate Flux in Crossflow Ultrafiltration under Intermediate Pressure. J. Colloid and Interface Sci. 214: 251-263.

11] Bowen, W.R., A.W. Mohammad, and N. Hilal 1997. Characterisation of Nanofiltration Membranes for Predictive Purposes-use of Salts, Uncharged Solutes and Atomic Force Microscopy. J. Membrane Sci. 126: 91-105.

[12] Nagy, E. Mass Transport in Biocatalytic Membrane Reactor, CHISA'08. 24-28 Aus. 2008. Prague, Czehrepublic. 1509. pdf

[13] Schafer; T., and J.G. Crespo. 2007. Study and Optimization of the Hydrodynamic Upstream Conditions during Recovery of a Complex Aroma Profile by Pervaporation . Membrane Sci. 301: 46-51.

\section{APPENDIX}

The concentration distribution obtained by the integration of Equation (4) can be given by Equations (5) and (6). The values of parameters, $T_{L}, Q_{L}, T_{m}, Q_{m}$ can be obtained by the solution of the algebraic equations using the internal (7) and (1 pas transfer rate, concentration polarization modulus, enrichment, the concentration profiles in both the boundary and membrane layers, etc. can be calculated. For this prediction all important transport parameter; namely $P e_{L}, P e_{m}, S_{m}$, should be known.

$$
\begin{gathered}
T_{L}=\frac{C_{b}\left(1-e^{-P e_{m}}-S_{m}\right)+S_{m} C_{p} e^{-P e_{m}}}{S_{m} e^{P e_{L}}-e^{-P e_{m}}+1-S_{m}} \\
Q_{L}=S_{m} \frac{C_{b} e^{P e_{L}}-C_{p} e^{-P e_{m}}}{S_{m} e^{P e_{L}}-e^{-P e_{m}}+1-S_{m}}
\end{gathered}
$$




$$
T_{m}=S_{m} \frac{-C_{b} e^{P e_{L}}+C_{p}\left(S_{m} e^{P e_{L_{L}}}+1-S_{m}\right)}{e^{\left[P e_{m}\left(1+\delta / \delta_{m}\right)\right]}\left(S_{m} e^{P e_{L}}-e^{P e_{m}}+1-S_{m}\right)}
$$

$$
Q_{L}=Q_{m}
$$

where $P e_{L}=v \delta / D_{L}, P e_{m}=v \delta_{m} / D_{m}$.

Equations (A1) to (A4) give the concentration distribution, in the direction of the mass flow, i.e. perpendicular to the membrane interface, in a local position of the membrane. The mass transfer rate equation, Equation (15) is valid also in a local position. This mass transfer should then be replaced into the boundary conditions of the differential balance equation given for a capillary membrane, in axial direction. In this case the value of $C_{b}$ and $C_{p}$ can be a function of the axial coordinate. 